\title{
The Effects of a Bioenergy Economy Based Program on Attention Bias Modification in People With High Anxiety Sensitivity
}

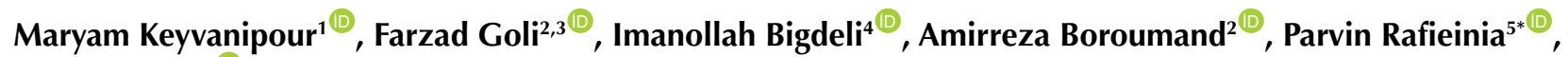 \\ Parviz Sabahi ${ }^{5}$ (1)
}

'Department of psychology, Faculty of Education and Psychology, Semnan University, Semnan, Iran

${ }^{2}$ Danesh-e Tandorosti Institute, Isfahan, Iran

${ }^{3}$ Professor, Energy Medicine University, California, United States

${ }^{4}$ Professor of Psychology, Faculty of Education and Psychology, Ferdowsi University of Mashhad, Mashhad, Iran

${ }^{5}$ Assistant Professor of Psychology, Faculty of Education and Psychology, Semnan University, Semnan, Iran

\begin{abstract}
Background: According to scientific evidence, high levels of sensitivity anxiety are one of the predictors of panic attacks, anxiety, and depression. The multidimensional anxiety sensitivity (cognitive, social, and physiological) is base on cognitive biases such as attention biases which are due to selecting threatening stimuli instead of neutral or positive stimuli. Despite the initial promise, attention bias modification ( $\mathrm{ABM}$ ) has a limited effect on reducing anxiety. This study aims to modify attention bias by reducing the focus on the threatening stimuli based on Bioenergy Economy (BEE) protocol; as an integrated model of care.

Methods: The present study is base on a quasi-experimental design with pre-test and post-test and follow-up in both groups. Thirty women between the ages of 23 to 50 selected from the patients referred to Bozorgmehr Neurology clinic in Mashhad. People who scored more than 70 in anxiety test, were randomly put into the experimental and control groups. The data collection tool was dotprobe test. The experimental group participated in the entire BEE protocol.

Results: The mean score sensitivity anxiety of the experimental group in the pre-test, post-test, and follow-up was significantly lower than those in the control group by using multivariate analysis of covariance. Also, the mean score of the experimental group in dot-probe test, neutral reaction time, emotional response time, and interference score in the post-test phase, as well as the components of dull reaction time and sensitive response time in the follow-up and post-test were significantly lower than the control group.

Conclusion: The BEE protocol is effective in ABM and reducing high sensitivity anxiety.

Keywords: Attention bias, Sensitivity, anxiety, Bioenergy Economy.
\end{abstract}

\author{
*Correspondence to \\ Parvin Rafieinia, \\ Assistant Professor of \\ psychology, Faculty of \\ Education and Psychology, \\ Semnan University, Semnan, \\ Iran. \\ Email:p_rafieinia@semnan.ac.ir
}

Published online September 23, 2019

Citation: Keyvanipour M, Goli F, Bigdeli A, Boroumand A, Rafienia P, Sabahi P. The effects of a bioenergy economy based program on attention bias modification in people with high anxiety sensitivity. Int Clin Neurosci J. 2019;6(4):133-139. doi:10.15171/icnj.2019.25.

\section{Introduction}

Anxiety disorders, including panic disorder with or without agoraphobia, generalized anxiety disorder, social anxiety disorder, specific phobias, and separation anxiety disorder, are the most prevalent mental disorders and are associated with enormous health problems. ${ }^{1}$ Instead of using the popular term fear to fear, which can mean many different things to different people, Reiss and McNally precisely defined the concept, which they called anxiety sensitivity (AS). This is the fear of anxiety-related bodily sensation, arising from the person's beliefs about the dangerousness of sensation. The bodily sensation associated with anxiety includes rapid heartbeat, sweating, trembling, dizziness, and concentration difficulties. Reiss and McNally proposed that people differ in their levels of
AS. Some people have little or no AS, some people have extremely high levels, and some have moderate levels. ${ }^{2,3}$ People with high levels of AS tend to be very frightened of anxiety-related sensations. If they experience a rapid heartbeat, they are likely to believe that they are going to die of a heart attack. If they sweat to tremble in public, they are likely to worry that people will reject or ridicule them. If they have trouble concentrating because they are anxious, they worry that their concentration difficulties will lead to insanity. ${ }^{4}$ Panic disorder and panic attacks have associated with selective attention to threatening information, a factor that may contribute to the maintenance of panic. AS, or fear of anxietyrelated symptoms, has been described as a cognitive risk factor for panic. It is of interest to understand the 
relationship between these cognitive correlates of panic, but the literature on the topic has been equivocal. In a study, 65 individuals completed measures of AS, anxiety symptoms and panic as well as the emotional Stroop task, a commonly used measure of attentional bias. We found that panic history and anxiety symptoms were associated with an attentional bias for information related to physical and social threat. ${ }^{5}$

\section{Origins of Anxiety Sensitivity}

AS is associated with an attentional bias for painrelated information in healthy children and adolescents. According to MacLeod \& Mathews, one such potential intervention is attention bias modification (ABM), a computer-delivered treatment for anxiety that emerged from research in experimental psychopathology. ${ }^{6}$ The goal of $\mathrm{ABM}$ is to reduce anxiety by reducing attention bias (AB) towards the threat, which refers to the preferential tendency to allocate attention to threatrelated information rather than nonthreat information. Despite initial promise, ABM has limited effectiveness in reducing anxiety. ${ }^{7,8}$ Anxiety-linked attentional bias and its modification: illustrating the importance of distinguishing processes and procedures in experimental psychopathology research. It is important to examine the cognitive mechanisms underlying $\mathrm{AB}$ in anxiety ${ }^{9}$ to improve $\mathrm{ABM}$.

\section{Attention Bias Modification}

Highly anxious individuals tend to demonstrate an attentional bias for threatening information and stimuli. To mitigate these biases and reduce anxiety symptomatology, attention bias modification programs (ABMPs) have been developed to train attention away from threat using modified dot-probe tasks. Recent efforts within the literature have focused on tailoring the methodology and experimental protocols of these tasks to increase the efficacy of training, with limited attention given to the impact of patient characteristics on one's amenability to such practice. A feature known to influence recognition and information processing is attachment style. Therefore, as an example, there is a study which is performed an ABMP and assessed the moderating effects of attachment style on training effectiveness among 126 college students from Biola University. ${ }^{10}$

The presence of attention biases in AS indicates the feasibility of treatment by reducing the negative attention biases associated with body sensations and improving the symptoms of the disorder. Through the conscious leading of attention and integrating attention, which is the economic attention of bioenergy economy (BEE), we can reduce body sensations attention bias and improve AS. Since BEE is a non-medical and harmless treatment, it has recommended as an effective way to reduce anxiety in patients and healthy people.
Bioenergy Economy

$\mathrm{BEE}$ is an integrative, evolutionary, body-centered approach to care. Releasing blockages, reprocessing energy information flows, resonating biofield, and opening the whole body to being are the main strategies of this meta diagnostic approach. The main goal of the BEE is sustainable development of happiness. This care system tries to integrate matter-energy-informationconsciousness process through four levels; body economy, narrative economy, relation economy, and intention economy. BEE works on the embodied aspect of experience among intrapersonal (physical, and energetic), intracorporal, and transpersonal bodies. ${ }^{11}$

For this purpose, bodywork, energy work, mindful, and psychodynamic techniques employed in an integrative manner. Body economy, the first level, focuses on mindful cathexis of physical body and harmonization of tones of tactile, proprioceptive, vestibular, and visceral perceptions. Body economy leads to the development of grounding and "tensegrity" state. Development of body awareness promotes self-awareness and security. The sense of tensegrity is not only our state of automatic tuning but a reference point which can be returned to when any chaos occurs in thinking and emotions. By re-establishing tensegrity and whole-body experience, without dealing with mind content, we can regulate our emotions. Having the body tuned, when we come back to the same thoughts and feelings, we often find out there exists no such problem to be solved, the problem changes or becomes clearer with sharp confines and illustrations. ${ }^{12}$

The second level is the narrative economy. Through deepening our understanding of the physical body, we can percept subtler body sensations; energetic body. Our touchstone of being at this level is "fluent-centered state" - the feeling of being fluent while concentrated in the center of gravity of the body. In the last few decades, instruments to measure electromagnetic fields and streams in the body have developed, and diagnostic and therapeutic methods based on the employed to a great extent. Electromagnetic flows not only spread all over the body through veins and nerves but also through the more extensive connective tissue system called the living matrix. Physical and emotional traumas and chronic stressors can cause blockages in this network leading to biological and psychological dysfunctions. ${ }^{13}$

The relation economy, the third level, is based on integrating interpersonal cathexis. From energy economy perspective, the relationship is symbolic and spatiotemporal extensions of the body and interaction of our phenomenal field with other fields. Through sharing its resources and boundaries, our biofield can reach higher dynamic-kinetic sustainability. The relationship is not mere interaction but is participation in more complex systems. For the more sustainable connection between self and other, we have to go beyond imaginary and rigid limits of mental self and understand a person not as an object 
with a name but as a reflective communicative action in space-time. In this level, consciousness transcends natural boundaries of the skin to the communicative proxemics of ours, and our body becomes a mindful biofield. Whether we are aware of current communicative bodies or not, our body parts participate in larger intracorporal systems; although we conceptualize all those complex multidimensional experiences only in the frame of egoistic narratives. The fourth level, intention economy, is related to the nonlocal body and transpersonal experiences. Consciousness emerged from matter, but it is nonlocal. Although mindfulness can identify with anywhere and anything, it cannot be known where in place it is located. The organism which is aware of its existence or, in Heidegger's words, Dasein, cares for being and makes "beings" express "the being."

The body extends not only in domains of intrapersonal and interpersonal relationships but into the area of transpersonal relationships. The transpersonal realm includes the relationship of the body with the whole. Conscious body simultaneously is in relation with self, other, and the whole although in any time inclines to concentrate on one of these dimensions. Transpersonal experience is to derive security of boundarylessness and, in Ungaretti words, m'illumino d'immenso. This is not a regressive metamorphosis which wipes out individuality but is the experience of unos-ambo or dual unity. It is the individuality which is rooted in existence, not out of it. The uniqueness beyond the origin of paranoiac ego is not a mental experience, but body awareness. ${ }^{14}$

The principal goals of BEE intervention are to increase the coherence of energy-information flow and selforganization of the body via the development of body awareness, increasing mind-body coordination, and modification of lifestyle. The current study aims to modify attention bias by reducing the focus on the threatening stimuli based on BEE protocol; as an integrated model of care.

\section{Materials and Methods}

The revised AS index is a 36-item self-reporting tool developed by Taylor et a ${ }^{15}$ to measure the fear of symptoms associated with anxiety. This tool is specially designed to evaluate and measure hierarchical structure, consisting of a factor analysis study, concluded that the revised index of AS has a hierarchical structure. These factors include (1) Fear of respiratory symptoms, (2) fear of cognitive dyscontrol, (3) fear of gastrointestinal symptoms, and (4) fear of cardiac symptoms. These loaded on a single higher-order factor. Respondents show their agreement with each of the matters on a Likert scale that extends from the "very low" to "extensive" range. The scores range from zero to 144, respectively, indicating the lowest and highest ratings.

The results of Taylor et $\mathrm{al}^{17}$ show that the revised index of AS was calculated based on 3 methods of internal consistency, re-test, and sub-sampling practice. For the full scale, the coefficients of reliability were $0.93,0.95,0.97$ achieved. Also, the reliability coefficients of the subscales were calculated based on the internal consistency, re-test, and sub-sampling method, which ranged from 0.82 to $0.92,0.92$ to .96 , and to 0.76 to 0.90 , respectively.

\section{Visual Dot-Probe Test}

Visual Dot-Probe test presented by MacLeod et al. ${ }^{15}$ In this test, instead of words, facial images have been used with various emotional states (angry, happy, and neutral). In this test, each of angry, happy, and neutral emotional faces (as large as $1.8 \times 2.5 \mathrm{~cm}$ ) paired with an image of a neutral sensitive face. Pictures and dots presented in two rectangular boxes with a length of $3.5 \mathrm{~cm}$ and a width of $3 \mathrm{~cm}$ and at a distance of $2 \mathrm{~cm}$ from the central point of the screen, each image is presented 40 times, 20 times on the right and 20 times on the left, and the position of the location is also determined based on contrast repetition. The subject placed at a distance of $50 \mathrm{~cm}$ from the computer screen. First, an empty box and a stabilization point (+) presented for 500000 seconds. Then, there are two faces on the left and right of the stabilization point for 250000 seconds on the screen. After that, the image of the emotional faces provided for 50000 seconds is covered. Then, a point replaces one of the pictures. The subject must press the arrow keys on the computer keyboard and, accordingly, the computer records the subject's reaction time to one-thousandth of a second. Altogether, 200 significant attempts and ten attempts to practice and familiarise with the test conducted for each subject. ${ }^{17}$ The results of dot-probe test in social anxiety indicate the differential validity of this test and its ability to evaluate attention bias. $^{16}$

\section{The Bioenergy Economy Program}

The educational and clinical bioenergy intervention was used, which was based on Bioenergy Economy Program in California Energy Medicine University in the United States, by Goli et al. ${ }^{17}$ The program aims to integrate the body in four-level; physical (body), energy economy, narration, and relation levels. Mass and energy economy named as body economy, and the narration and relationship are called a narration economy in training sessions. For this purpose, some information about the body training, strategies, and training techniques presented to the participants.

\section{Participants and Methods}

The present study is a quasi-experimental design with pretest and post-test and follow-up in the control group. The statistical population of the study included all women aged 23 to 50 years old who referred to Bozorgmehr Neurology Clinic of Mashhad in Jan-Feb of 2016. Among the referred patients, 30 people were selected and randomly put into 2 groups of the experimental and control group. 
The present study was conducted as a protocol of 6 sessions totally and a session per week (160 minutes) educational and clinical training, every course includes 30 minutes rest time, and it has based on BEE program. Subjects repeated the exercises during the week at home. Also, after each session, the text of the practices presented as a flashcard and a reminder on the telegram channel. At the end of each session, the audio file of methods and the order of the work immediately provided to the participants. Physical exercises examined through the emotional feedback of the participants, and their effect was on material changes in individuals. During each activity, participants have given specific instructions on how to experience the theme through their bodies, followed by a guided meditation on their bodies, thoughts, and emotions. The regular practice of the task included the replication at home at least once every day; more free training occurred in real-life settings as the nature of the responsibility and opportunity determined. Participants were also asked to express their thoughts and feelings during the week at the beginning of each session. The primary purpose of these feedbacks was to increase body awareness, thought, and feeling. Notably, participants were asked to stay aware and report any changes in their personal life.

At first, the participants randomly selected, then; a pretest, which included dot-probe test and AS test, was taken from the subjects and at the sixth session, all subjects were retested again (as the post-test). A followup test conducted for three months (90 days) after completing the training sessions. The contents of the courses summarised below;

First session: Familiarity with the program and group members; pre-test; the role of anxiety in health; strong economy, body-emotion-thought cycle; the importance of the body in emotion regulation, relations, and cognition.

Techniques; tensegrity or (grounding) and body awareness in the formal and informal state. Second session: BEE program; cathexis and satisfaction development; activation of health and self-regulation of the system, trust on the organism, the body consciousness, the importance of awareness in preventing reaction and releasing tension. Techniques; body awareness (formal and informal). Third session: Familiarity with body memory; Integrity in the fluidity of consciousness on timely or untimely cathexis, energy blockers, and non-economic cathexis, energy cycles, familiarity with attunement. Fourth session: Week experiences review; familiarity with turning, conscious attention guide, inner and outward attention guide, economic attention, the importance of gratitude exercises. Fifth session: Familiarity with narrating, Centrality in fluidity; Making a narrative for life, self-narration, the importance of body integrity and safety in narration, grudge, and blame in the story of non-life, hell car, Importance if forgiveness; self-care is the secret of life. Sixth session: Review of the experiences during the past three weeks; Setting a daily schedule, understanding clues, and using keys to make a better life. Review of body awareness, attunement and narrating, giving some clues in body, attention, and thought that represent a non-economic orientation.

\section{Results}

The mean (SD) age for the experimental group was 46.33 (8.7), and for the control group, it was 38.32 (7.5). Among 15 participants in the experimental group, most of them have a bachelor degree ( 8 students). Also, the 13 participants in the control group who participated in this study had the highest number in bachelor degree (10 students). Based on the results presented in the table, the mean and standard deviation of the AS scores in the pre-test were 70.93 and 25.57, in the post-test, respectively, 37.13 and 34.57 , in follow-up respectively, and 20.05 and 25.42. For the control group, the mean and standard deviation of the sensitivity anxiety scores in the pre-test were respectively 76.23 and 21.26 , in the post-test were respectively 75.46 and 31.71 , and in followup were 66.54 and 34.68 . Based on the results for the experimental group, the mean of neutral reaction time, emotional response time and interference score in the pre-test were respectively $822.85,823.37$ and 0.52 , and in the post-test, respectively, 475.19, 477.82 and 2.63; and in the follow-up phase respectively, 470.72, 474.12, and 3.39. For the control group, the mean of neutral reaction time, emotional response time and interference score in the pre-test were respectively $612.59,614.79$ and 2.19 , in the post-test were respectively $544.79,558.48$ and 13.69 . And in the follow-up were 577.65, 606.51, and 28.85 .

The first question of the research stated whether a BEE program affected the AS of people with anxiety symptoms? To answer this question, a multivariate covariance analysis used. In this analysis, the groups (experimental and control group) as inter-subject factors, AS scores in the pre-test as a control variable and the scores of this variable included in post-test and follow-up as dependent variables.

The results of the multivariable co-variance analysis in Tables 1 and 2 show that there is a significant difference between the two experimental and control groups in the new variable that results from the linear combination of post-test and follow-up scores of AS as dependent variables (Wilks' lamdba $=61.0, P<0.01, \mathrm{~F}=6.69$ ).

In order to compare the 2 groups for the AS scores in each of the measurement steps, the results of the test between the subjects in Table 2 show that among the participants in the both groups, the mean scores of AS, both in the post-test phase $(P<0.01)$ and follow up $(P<0.001)$.

Despite the descriptive index in Table 2, this difference in post-test and follow-up shows that the experimental group means scores are significantly lower than the mean scores of the control group. 
Table 1. Multivariate Analysis of Covariance Results for Sensitivity Anxiety Scores

\begin{tabular}{lccccc}
\hline Effect & Value & df Hypothesis & df Error & F & P Value \\
\hline Wilks Lambda & 0.61 & 2 & 21 & 6.69 & 0.006 \\
\hline
\end{tabular}

Table 2. Test Results of the Effects Among the Cases to Compare the Sensitivity Anxiety Scores of the 2 Groups

\begin{tabular}{lccccc}
\hline Statistical Variable Index & Sum of Squares & df & Mean of Squares & F & P Value \\
\hline Sensitivity anxiety (post-test) & 7901.65 & 1 & 7901.65 & 8.00 \\
Sensitivity anxiety(follow-up) & 10343.94 & 1 & 10343.94 & 14.02 \\
\hline
\end{tabular}

The second question of the research stated whether a BEE program affected the attention biases of people with anxiety symptoms? To answer this question, a multivariate covariance analysis used. In this analysis, the groups (experimental and control group) as inter-subject factors, AS scores in the pre-test as a control variable and the scores of this variable included in post-test and follow-up as dependent variables.

The results of multivariable covariance analysis in Table 3 show that there is a significant difference between the two experimental and control groups in the new variable which results from the linear combination of post-test scores and follow-up of neutral response time, emotional response time and interference score as dependent variables (Pillai's trace $=0.53, P<0.01, \mathrm{~F}=5.08$ ).

In order to compare the experimental and control groups for the components of Dot probe test at different stages of the measurement, the results between the subjects in Table 4 show that among the participants of the two groups, three components of the dot-probe test, time negative reaction $(P<0.01)$, emotional reaction time $(P<0.001)$ and interference score $(P<0.05)$ in the posttest, as well as in the reaction time neutral component $(P<0.05)$ and emotional response time $(P<0.05)$ in follow-up. It means there is a significant difference in the post-test and follow-up phase.

Despite descriptive indexes in Table 4, this difference in post-test and follow-up shows that the experimental group means scores are significantly lower than the mean scores of the control group.

\section{Discussion}

The results of this showed that there is a significant difference between the participants in the experimental and control groups in the mean scores of AS, both in the post-test and follow-up. The difference shows that the mean scores of the experimental group are significantly lower than the mean scores of the control group. These results indicate that the $\mathrm{BEE}$ protocol is effective in reducing sensitivity anxiety. These results are consistent with the findings of previous research on the effects of BEE program on some psychosomatic and congenital illnesses: For example; In a study Derakhdha et al. ${ }^{18}$ The findings of the study, Goli and Boroumand, ${ }^{17}$ refer to the effects of a brief BEE program on a patient with tethered cord syndrome. Goli in Bioenergy Economy book and Bioenergy Economy: A Biosemiotic Model of Care research introduced described the steps and method of its effect, which is consistent with the results of this study. Ahangar Ahmadi et $\mathrm{al}^{19}$ showed the consequence of awakening teachers to their presence through body awareness according to BEE Program. Also, the mean score of the experimental group in each three components of dot-probe test, neutral reaction time, emotional

Table 3. Multivariate Analysis of Covariance Related to the Scores of Dot-Probe Components

\begin{tabular}{lccccc}
\hline Effect & Value & $\boldsymbol{d f}$ Hypothesis & df Error & F & $\boldsymbol{P}$ Value \\
\hline Pillai's trace & 0.53 & 4 & 18 & 5.08 & 0.006 \\
\hline
\end{tabular}

Table 4. Results of Between Subjects Effects

\begin{tabular}{|c|c|c|c|c|c|}
\hline Variable Statistical Index & Sum of Squares & $d f$ & Mean of Squares & $\mathbf{F}$ & $P$ Value \\
\hline Neutral reaction time (post-test) & 84193.46 & 1 & 84193.46 & 9.68 & 0.005 \\
\hline Emotional reaction time (post-test) & 170860.34 & 1 & 170860.34 & 13.37 & 0.001 \\
\hline Interference score (post-test) & 15176.13 & 1 & 170860.34 & 13.37 & 0.024 \\
\hline Neutral reaction time (follow-up) & 70247.55 & 1 & 70247.55 & 6.23 & 0.021 \\
\hline Emotional reaction time (follow-up) & 141139.6 & 1 & 141139.16 & 6.62 & 0.018 \\
\hline Interference score (follow-up) & 12241.73 & 1 & 12241.73 & 3.48 & 0.07 \\
\hline
\end{tabular}


response time, and interference score in the post-test phase, as well as the elements of dull reaction time and sensitive response time in the follow-up and post-test were significantly lower than the participants of control group. In previous studies, the effect of a program based on body-mind control and energy medicine on cognitive variables, such as attention has not found.

According to the mechanisms of the BEE program, as discussed above, how these mechanisms affect the anxiety and attention bias can be explained as follows:

Body awareness and development of grounding and "tensegrity" state lead to releasing blockages, reprocessing energy information, and anxiety adjustment. So, it can make our behavior more flexible. On the other hand, energy exercises, without interfering in the complexity of the conceptualized mind, organize the emotions directly by organizing the mind and reprocessing body energy. Forgiveness exercises change the bioenergy orientation in the intrapersonal and interpersonal world, and it also turns reproduction of pain and anger to others, extends self-care and its boundaries from the intrapersonal to the interpersonal and transpersonal world. This changing from others to yourself and from its devastation to creation helps not only organize the emotions, which can make the boundaries broader and more flexible. Energy rotation from the object to the body; which is the main principle of BEE; can cause flexibility in deconditioning cathexis on the purposes. Because the cathexis on objects is based on the recognition of the object and previous experience, while body care and presence experience occur now. Conscious attention focus leads to change attention from inactive unconsciousness, obsession, and stressful stimuli to positive stimuli and more economic cathexis. Also, gratitude exercises and shifting attention from barriers to resources cause changes in the phenomenal field, the organization of emotions, and the economic responses.

Durability and sustainability of the intervention effect in the follow-up phase can be due to 2 reasons: Firstly, the basis of this method is to activate the person and his role. It can say that the sense of control has sustained effects. Secondly, the coordination of the change in the contextual body experience with the change in cognition can make the intervention lasting, because its music has changed attunement to cognitive change. Individual cognitive change alone doesn't have such an effect, because the person's emotions effect on the attention and cognitive biases, and thus the level of his AS will still be affected.

It has recommended that follow-up studies added to the research project for six months and one year after the intervention to measure the sustainability of the therapeutic effects. This intervention effect on biological and physiological indices associated with AS also should be investigated. On the other hand, BEE interventions should compare with other psychological interventions. Also, $\mathrm{BEE}$ interventions can apply in psychiatric disorders and other cognitive disorders such as CHDT, cardiovascular disease, depression, and obsessive disorder.

\section{Conflict of Interest Disclosures}

The authors declare that they have no conflict of interests.

\section{Ethical Statement}

In this study, informed consent was obtained from all participants and confidentiality of information was emphasized.

\section{References}

1. Bandelow B, Michaelis S. Epidemiology of anxiety disorders in the 21st century. Dialogues Clin Neurosci. 2015;17(3):32735.

2. Reiss S, Peterson RA, Gursky DM, McNally RJ. Anxiety sensitivity, anxiety frequency and the prediction of fearfulness. Behav Res Ther. 1986;24(1):1-8.

3. Allan NP, Capron DW, Raines AM, Schmidt NB. Unique relations among anxiety sensitivity factors and anxiety, depression, and suicidal ideation. J Anxiety Disord. 2014;28(2):266-75. doi: 10.1016/j.janxdis.2013.12.004.

4. Stewart S, Taylor S, Watt M. Overcoming the fear of fear: How to reduce anxiety sensitivity. Oakland, CA: New Harbinger Publications; 2007.

5. Lang AJ, Sarmiento J. Relationship of attentional bias to anxiety sensitivity and panic. Depress Anxiety. 2004;20(4):190-4. doi: 10.1002/da.20030.

6. Herron-Marx S, Price-Knol F, Burden B, Hicks C. A systematic review of the use of Reiki in health care. Altern Complement Ther. 2008;14(1):37-42. doi: 10.1089/act.2008.14108.

7. Heeren A, Mogoase C, Philippot P, McNally RJ. Attention bias modification for social anxiety: A systematic review and meta-analysis. Clin Psychol Rev. 2015;40:76-90. doi: 10.1016/j.cpr.2015.06.001.

8. Klauke B, Deckert J, Zwanzger P, Baumann C, Arolt $\mathrm{V}$, Pauli $\mathrm{P}$, et al. Neuropeptide $\mathrm{S}$ receptor gene (NPSR) and life events: $G \times E$ effects on anxiety sensitivity and its subdimensions. World J Biol Psychiatry. 2014;15(1):17-25. doi: 10.3109/15622975.2011.646302.

9. Mogg K, Bradley BP. Anxiety and attention to threat: Cognitive mechanisms and treatment with attention bias modification. Behav Res Ther. 2016;87:76-108. doi: 10.1016/j. brat.2016.08.001.

10. Siefke JJ. Attachment as a moderator of attention bias modification effectiveness. Biola University; 2015.

11. MacLeod C, Grafton B. Anxiety-linked attentional bias and its modification: Illustrating the importance of distinguishing processes and procedures in experimental psychopathology research. Behav Res Ther. 2016;86:68-86. doi: 10.1016/j. brat.2016.07.005.

12. Zvielli A, Bernstein A, Koster EH. Temporal dynamics of attentional bias. Clin Psychol Sci. 2015;3(5):772-88.

13. Goli F. Bioenergy Economy: A Biosemiotic Model of Care. International Journal of Body, Mind and Culture. 2016;3(1):17.

14. Collinge W, Wentworth R, Sabo S. Integrating complementary therapies into community mental health practice: an exploration. J Altern Complement Med. 2005;11(3):569-74. doi: 10.1089/acm.2005.11.569.

15.

16. Taylor S, Zvolensky MJ, Cox BJ, Deacon B, Heimberg RG, Ledley DR, et al. Robust dimensions of anxiety 
sensitivity: development and initial validation of the Anxiety Sensitivity Index-3. Psychol Assess. 2007;19(2):176-88. doi: 10.1037/1040-3590.19.2.176.

17. Putwain DW, Langdale HC, Woods KA, Nicholson LJ. Developing and piloting a dot-probe measure of attentional bias for test anxiety. Learn Individ Differ. 2011;21(4):478-82. doi: 10.1016/j.lindif.2011.02.002.

18. Goli F, Boroumand AR. Back to Future Health Blueprint: The Effects of a Brief Bioenergy Economy Program on a Patient with Tethered Cord Syndrome. International Journal of Body,
Mind and Culture. 2016;3(1):64-9.

19. Derakhshan A, Manshaei G, Afshar H, Goli F. Effect of a bioenergy economy program on pain control, depression, and anxiety in patients with migraine headache. International Journal of Body, Mind and Culture. 2016;3(1):30-45.

20. Ahangar Ahmadi S, Henning JE, Goli F. Awakening Teachers to their Presence: An Experiential Course in Body Wisdom. Journal of Counseling and Professional Psychology. 2017;6(1):92-107. 